\title{
DENSIDADE ENERGÉTICA DAS REFEIÇÕES OFERECIDAS PELO SERVIÇO DE NUTRIÇÃO E DIETÉTICA DE UM HOSPITAL
}

\author{
Patrícia Bergjohann ${ }^{1}$, Fernanda Scherer Adami², Patricia Fassina ${ }^{3}$
}

\begin{abstract}
Resumo: A Densidade Energética pode ser utilizada na recuperação do estado nutricional, já que a desnutrição é comum em indivíduos hospitalizados. O objetivo do estudo foi verificar a $\mathrm{DE}$ de sete dias das refeições oferecidas pelo Serviço de Nutrição e Dietética de um hospital do interior do Rio Grande do Sul. A DE das preparações foi obtida pelo quociente do Valor Energético Total pelo volume (g) e classificada segundo o National Center for Chronic Disease Prevention and Health Promotion, 2005. Para a análise estatística utilizou-se o software Statistical Package for the Social Sciences, adotando-se o nível

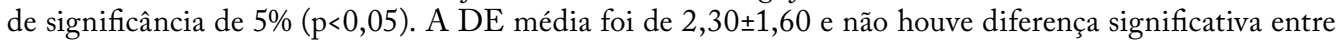
os dias, mas entre refeições e tipos de dieta. A média da DE encontrada indicou um bom planejamento de cardápios, porém as diferenças significativas entre as refeições e as dietas sugerem uma revisão dos cardápios.
\end{abstract}

Palavras-chave: Dieta. Ingestão de energia. Serviço de alimentação. Refeições.

\section{ENERGY DENSITY OF MEALS OFFERED BY A SERVICE OF NUTRITION AND DIETETICS FROM A HOSPITAL}

\begin{abstract}
Energy density (ED) can be used in the recovery of the nutritional status, since malnutrition is common in hospitalized individuals. The present study, in which it was found the daily during seven days of the five meals offered by Nutrition and Dietary Service of a hospital on the interior of Rio Grande do Sul. Energy density was calculated by dividing the Total Energy Value of Preparation by the weight (g) of each preparation and ranked ED according to the National Center for Chronic Disease Prevention and Health Promotion, 2005. Statistical analysis was performed using Statistical Package for the Social Sciences software, with a significant level of $5 \%(p<0,05)$. The average was $2.30 \pm 1.60$, and there was no significant difference between the DE day of the week, but between meals and diets. The average found $\mathrm{DE}$ indicates good planning menus, but significant differences suggest a revision in the planning of menus.
\end{abstract}

Keywords: Diet. Energy Intake. Food Services. Meals.

1 Acadêmica do Curso de Nutrição - Centro Universitário Univates, Lajeado-RS, Brasil.

2 Professora Doutora do Curso de Nutrição - Centro Universitário Univates, Lajeado-RS, Brasil.

3 Professora Mestre do Curso de Nutrição - Centro Universitário Univates, Lajeado-RS, Brasil. 


\section{INTRODUÇÃO}

Em termos de denominação, os serviços que atendem coletividades recebem a denominação de Serviço de Alimentação e Nutrição (SAN) e Serviço de Nutrição e Dietética (SND) quando relacionado à população enferma (AGUIAR; KRAEMER; MENEZES, 2013). Já Teixeira et al. (2010) denominaram esses serviços como Unidades de Alimentação e Nutrição (UAN), considerando que hospitais e centros de saúde promovem atividades-fim e o serviço que atende comensais de empresas, escolas e demais organizações é caracterizado como atividade meio, e conforme Aguiar, Kraemer e Menezes (2013), o termo Unidade Produtora de Refeições (UPR) vem sendo utilizado para todos os serviços inseridos na alimentação fora do lar, tanto comerciais, quanto coletivos.

Nas UANs hospitalares a densidade energética pode ser utilizada como uma forma de terapia tanto para tratar casos de obesidade e outras doenças crônicas como auxiliar na recuperação do estado nutricional e no processo de ganho de peso, já que a desnutrição é comum em indivíduos hospitalizados, decorrente da condição clínica aliada à aceitação da dieta hospitalar, assim como o estado psicológico prejudicado e mudança de hábitos (OLIVEIRA et al. 2010). Para que a DE estabeleça uma relação positiva com as UANs hospitalares, a avaliação e o acompanhamento do estado nutricional dos pacientes são imprescindíveis para a representação direta da DE na recuperação hospitalar (DIEZ-GARCIA; PADILHA; SANCHES, 2012), pois a DE pode ainda apontar e cooperar para uma redução no resto-ingesta do paciente hospitalizado, o qual representa o percentual de comparação entre a quantidade de refeição rejeitada e ofertada (FERREIRA; GUIMARÂES; MARCADENTI, 2013).

Desta forma, a DE possui estreita relação com a ingestão de energia e o balanço energético, principalmente em casos de inflamação sistêmica de pacientes hospitalizados em situação de câncer, como por exemplo (WALLENGREN; BOSAEUS; LUNDHOLM, 2013). Rodrigues e Proença (2011), em estudo de revisão concluíram que, conjugado à ingestão de energia e DE, está o tamanho da porção do prato, independentemente de idade, gênero e estado nutricional. Os mesmos autores inferiram ainda que o aumento no tamanho das porções é proporcional ao aumento da ingestão alimentar, sendo que esta se torna ainda maior mediante associação a uma maior DE. A ingestão alimentar pode ainda ser aumentada pela adaptação do cardápio às particularidades dos pacientes, podendo também reduzir o desperdício e o restoingesta através dessa estratégia (BARTON et al., 2000).

Conforme o National Center for Chronic Disease Prevention and Health Promotion (2005), a denominação da DE é expressa pela relação das calorias pelo peso da refeição (calorias por grama) e categorizada conforme o grau de densidade, assim, alimentos com alta densidade energética possuem um alto número de calorias em relação ao volume da refeição. Os alimentos de alta $\mathrm{DE}$ contêm elevado teor de gordura ou açúcar, como batatas-fritas, nozes, biscoitos, produtos cárneos e queijos. Já alimentos com uma DE reduzida, possuem mais água em sua composição, incluindo frutas, legumes e 
sopas (NATIONAL INSTITUTE FOR HEALTH AND CARE EXCELLENCE, 2015). Nas definições de Drewnowski et al. (2004), a densidade de energia dos alimentos é inversamente relacionada ao teor de água dos alimentos, proporcionalmente relacionada ao teor de gordura da dieta e positivamente relacionada à ingestão total energética. Através das classificações da DE também é possível constatar que esta possui associação com a qualidade da dieta (MENDES et al., 2016).

Há vários estudos indicando a $\mathrm{DE}$ tanto para o processo de perda de peso, como para a recuperação do estado nutricional em diferentes proposições de cálculos. Cox e Mela (2000) estudaram oito métodos distintos para calcular a DE, desde a inclusão de todos os alimentos e bebidas, até a exclusão de todos os líquidos. Wallengren, Lundholm e Bosaeus (2005) também analisaram vários métodos de cálculos e encontraram resultados indicando que o método utilizado para o cálculo da DE possui pouca influência sobre a relação entre a $\mathrm{DE}$ e diferentes consumos de energia.

O presente estudo objetivou verificar a $\mathrm{DE}$ de sete dias das refeições oferecidas pelo SND à população enferma, de um hospital do interior do Rio Grande do Sul e fornecer subsídios para a avaliação do planejamento de cardápios.

\section{METODOLOGIA}

Primeiramente foram selecionados aleatoriamente sete cardápios do mês de setembro do ano de 2015, incluindo cinco refeições, desjejum, almoço, lanche da tarde, jantar e ceia e os diferentes tipos de dietas, livre, pastosa e para diabetes mellitus, servidas no hospital para posteriormente calcular a densidade energética. O cálculo foi realizado dividindo-se o Valor Energético Total (VET) em quilocalorias (kcal) pelo peso total (volume) (g) de cada preparação utilizando-se o Software Microsoft Office Excel 2007.

As informações do VET e peso foram pesquisados nas fichas técnicas de preparação, documento que contém quantidade per capita de cada alimento, custo, peso da preparação pronta e modo de preparo. As preparações que não continham VET foram calculadas no Software DietWin Profissional versão 2008 e os alimentos tiveram seus rótulos verificados, quando necessário. Tanto os cardápios do hospital quanto as fichas técnicas foram disponibilizados pela nutricionista responsável do SND, sendo que as fichas técnicas de preparação foram elaboradas com base na Tabela Brasileira de Composição dos Alimentos (TACO, 2011).

Para as refeições estudadas foram considerados apenas os alimentos sólidos e leite, pois este produto lácteo representa uma possibilidade de maior aporte energético (RICARDO; CLARO, 2012; OLIVEIRA et al., 2010). A água foi excluída por não conter calorias, somente influenciaria no volume da preparação (ELLO-MARTIN; LEDIKWE; ROLLS, 2005). No desjejum efetuou-se o cálculo das dietas livre, pastosa e para Diabetes Mellitus, assim como no almoço. No lanche da tarde apenas a dieta livre, no jantar as dietas livre e pastosa e na ceia apenas a livre. 
Quando a DE resultou entre o valor de 4,0 e 9,0 kcal por grama de peso, a preparação foi categorizada como de alta $\mathrm{DE}$, quando entre 1,5 e 4,0 kcal por grama de peso como média $\mathrm{DE}$, quando de 0,7 a $1,5 \mathrm{kcal}$ por grama, de baixa $\mathrm{DE}$ e as preparações de muito baixa $\mathrm{DE}$ foram classificadas em uma faixa de zero a $0,6 \mathrm{kcal}$ por grama. A DE das preparações foi classificada de acordo com o National Center for Chronic Disease Prevention and Health Promotion (2005).

Os dados foram analisados no software Statistical Package for the Social Sciences (SPSS) da IBM ${ }^{\circledR}$, versão 20.0. O nível de significância adotado foi de $5 \%(\mathrm{p}<0,05)$. Foram realizadas estatísticas univariadas descritivas (médias, variâncias, desviospadrão e frequências) e bivariadas (testes de ANOVA e Qui-quadrado). Utilizou-se o teste de Kolmogorov-Smirnov para avaliar se as variáveis seguiam a distribuição normal. Como observou-se uma distribuição normal, os resultados foram analisados por testes paramétricos. O teste de ANOVA foi aplicado para comparar a densidade entre os dias da semana, tipos de dieta e refeições e o teste de qui-quadrado foi aplicado para avaliar a associação entre as categorias de densidade e os tipos de dieta e refeições.

\section{RESULTADOS}

A Tabela 1 mostrou que 48,3\% das preparações faziam parte do desjejum e 37,9\% do almoço. Destas preparações, $65,5 \%$ faziam parte de dietas livres, $31 \%$ dietas para

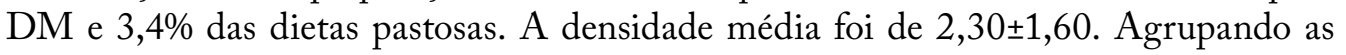
preparações em categorias, segundo a $\mathrm{DE}, 42,4 \%$ apresentaram média densidade, $21,7 \%$ muito baixa densidade, $21,2 \%$ baixa densidade e $14,8 \%$ alta densidade.

Tabela 1. Distribuição dos alimentos segundo a classificação da densidade, a refeição do dia e o tipo de dieta

\begin{tabular}{lcc}
\hline Densidade $[\boldsymbol{n}$ 203] & F & \% \\
\hline Muito baixa & 44 & 21,7 \\
Baixa & 43 & 21,2 \\
Média & 86 & 42,4 \\
Alta & 30 & 14,8 \\
\hline Refeição do Dia $[\boldsymbol{n}$ 203] & F & $\mathbf{\%}$ \\
\hline Desjejum & 98 & 48,3 \\
Almoço & 77 & 37,9 \\
Lanche da Tarde & 7 & 3,4 \\
Jantar & 14 & 6,9 \\
Ceia & 7 & 3,4 \\
\hline
\end{tabular}




\begin{tabular}{lcc}
\hline Tipo de Dieta $[\boldsymbol{n}$ 203] & F & \% \\
\hline Livre & 133 & 65,5 \\
Pastosa & 7 & 3,4 \\
DM & 63 & 31,0 \\
\hline
\end{tabular}

Resultados descritos em frequência absoluta (F) e percentuais (\%).

Fonte: dos autores.

A densidade foi significativamente maior $(\mathrm{p}<0,001)$ nas dietas livre $(2,56 \pm$ $1,57)$ e para $\mathrm{DM}(1,94 \pm 1,55)$ em relação à pastosa $(0,50 \pm 0,01)$, assim como foi significativamente maior $(\mathrm{p}=0,034)$ no lanche da tarde $(3,76 \pm 0,67)$ e na ceia $(3,38$ $\pm 1,10$ ) em comparação às demais refeições (desjejum: $2,28 \pm 1,55$; almoço: $2,10 \pm$ 1,72; jantar: $2,18 \pm 1,36$ ). Já comparando a densidade entre os dias da semana, não foi encontrada diferença significativa $(\mathrm{p}=0,993)$. As médias e valor de significância das comparações (p) podem ser conferidos na Tabela 2.

Tabela 2. Comparação da densidade energética dos alimentos segundo o tipo de dieta, refeição do dia e dia da semana

\begin{tabular}{lcc}
\hline Tipo de Dieta $[\boldsymbol{n}$ 203] & $\begin{array}{c}\text { Densidade Energética } \\
\text { Média } \pm \text { DP }\end{array}$ & $\mathbf{p}$ \\
\hline Livre & $2,56 \pm 1,57$ & \\
Pastosa & $0,50 \pm 0,01$ & $<, 001$ \\
DM & $1,94 \pm 1,55$ & $\mathbf{p}$ \\
\hline Refeição do Dia $[\boldsymbol{n}$ 203] & Densidade Energética \\
Média \pm DP & \\
\hline Desjejum & $2,28 \pm 1,55$ & \\
Almoço & $2,10 \pm 1,72$ & 0,034 \\
Lanche da Tarde & $3,76 \pm 0,67$ & \\
Jantar & $2,18 \pm 1,36$ & $\mathbf{p}$ \\
Ceia & $3,38 \pm 1,10$ & \\
\hline Dia da Semana $[\boldsymbol{n}$ 203] & Densidade Energética & \\
\hline Domingo & Média \pm DP & \\
Segunda & $2,28 \pm 1,50$ & \\
Terça & $2,34 \pm 1,53$ & \\
Quarta & $2,11 \pm 1,60$ & \\
Quinta & $2,39 \pm 1,59$ & \\
Sexta & $2,45 \pm 1,96$ & \\
Sábado & $2,26 \pm 1,61$ & \\
\hline
\end{tabular}

Fonte: dos autores. 


\section{DISCUSSÃO}

Evidências apontam a densidade energética como um indicador de qualidade da dieta, sendo alimentos como carnes magras, peixes, produtos lácteos com reduzido teor de gorduras, frutas e verduras classificados com menos energia por unidade de peso em relação à fast-foods, doces e sobremesas. Logo, dietas com baixa densidade energética, contendo alto teor de nutrientes, possuem relação com reduzido ganho de peso, menores índices de obesidade, diabetes mellitus tipo II, doença cardiovascular e alguns tipos de câncer (MONSIVAIS; DREWNOWSKI, 2009). A média da DE encontrada no presente estudo para indivíduos hospitalizados foi classificada como média $\mathrm{DE}$, os quais, dependendo dos casos individuais, necessitam de um aporte energético aumentado somado ao fato de apenas $14,8 \%$ das preparações avaliadas terem sido classificadas de alta $\mathrm{DE}$, havendo possibilidade de agravos ou lenta recuperação de uma parcela dos pacientes.

No estudo realizado por Stella (2008) com uma população entre 20 e 59 anos participante de um Inquérito de Saúde do estado de São Paulo, que excluiu todas as bebidas, foi encontrada uma DE média de $1,98 \mathrm{kcal} / \mathrm{g}$, indicando média DE. Resultado inferior ao atual estudo que observou uma $\mathrm{DE}$ média de $2,30 \mathrm{kcal} / \mathrm{g}$ realizado com pacientes hospitalizados e considerando o leite nos cálculos, fator que pode explicar a DE média do presente estudo ter sido superior em relação ao estudo citado.

Canella, Bandoni e Jaime (2011) estudaram a DE aplicada às empresas inscritas no Programa de Alimentação ao Trabalhador (PAT) e utilizou dois métodos distintos para determinar a DE: DE1 quando houve inclusão de todos os alimentos sólidos e bebidas, excluindo-se a água e DE2 quando houve inclusão de todos os alimentos sólidos e exclusão de todas as bebidas. Os autores encontraram médias de $1,10 \mathrm{kcal} / \mathrm{g}$ para DE1 e 1,43 kcal/g para DE2, sendo classificadas como baixa DE, ambas classificações inferiores à encontrada no presente estudo, as quais representam um fato preocupante, pois dependendo do trabalho que os referidos trabalhadores exercem, esta energia ofertada pode não suprir a energia gasta por eles, podendo acarretar na deficiência de nutrientes, a longo prazo pode comprometer a saúde dos trabalhadores e também pode haver uma redução no rendimento das suas atividades (ORO; HAUTRIVE, 2015). A diferença entre as médias de DE encontrada entre o estudo de Canella, Bandoni e Jaime (2011) e o presente estudo, pode ser explicada pela razão de que somente o cardápio do almoço de três dias foi analisado, enquanto que no presente estudo houve a análise de todas as refeições.

Em ambiente hospitalar, a dieta pastosa é utilizada para proporcionar repouso digestivo e fornece uma quantidade adequada de nutrientes, com uma consistência menos sólida, na forma de purês, papas, cremes, suflês e as carnes são moídas ou desfiadas. A dieta pastosa representa a transição entre a dieta semi-líquida e branda, sendo indicada para os casos de dificuldade de mastigação e deglutição, sendo geralmente ofertada para idosos, crianças pequenas, pacientes com patologias neurológicas, casos pós-operatórios e dispneia (CARUSO; SIMONY; SILVA, 2004). A média da DE da 
dieta pastosa do atual estudo classificou-se como muito baixa, resultado diferente ao estudo de Oliveira et al., (2010), no qual a média das preparações pastosas analisadas foram classificadas como baixa $\mathrm{DE}$, proporcionando assim, uma maior possibilidade de melhora na recuperação de pacientes. Visto a importância da alimentação em pacientes hospitalizados, vale destacar que o acréscimo de alimentos com uma DE maior, o modo de preparo e a utilização de suplementos nutricionais podem proporcionar um aumento da DE e oferecer uma contribuição na melhora do estado nutricional (SILVA, 2014).

Com o objetivo de manter o controle metabólico da glicose, destaca-se a dieta para DM, na qual há a preocupação de fornecer uma alimentação adequada em todos os nutrientes, com preferência por carboidratos complexos e fibras, na ocorrência de Diabetes Mellitus I e II (MARTINS et al., 2001). A média DE (1,94 kcal/g) encontrada nas dietas para DM do presente estudo foi um fator positivo para a patologia de DM, visto que a ingestão de alta densidade energética, acompanhada de um alto teor de gordura, pode provocar agravos como o sobrepeso e obesidade, com consequente resistência à insulina (LOTTENBERG, 2008). Por outro lado, na ocorrência de uma ingestão deficiente e baixa DE, é contraindicada a adição de açúcar, mas podese utilizar o acréscimo de leite às preparações, o que não causa alterações importantes no sabor (LEDIKWE et al., 2006). O presente estudo buscou avaliar a DE visando à melhora do estado do paciente hospitalizado, porém a maioria dos estudos encontrados sobre este tema tem seu foco na perda de peso e prevenção da obesidade em situações patológicas, como a DM.

A DE significativamente superior encontrada na dieta livre em relação à pastosa e para DM no presente estudo se justifica por ser ofertada para indivíduos sem necessidade de modificação em nutrientes e consistência. Logo, é o módulo dietético que inclui um maior número de alimentos, que sejam indicados em uma alimentação saudável, baseada na pirâmide dos alimentos (CARUSO; SIMONY; SILVA, 2004). Assim, uma DE mais elevada nesta dieta é explicada pelo fato de que não há restrição no tipo ou no método de preparo dos alimentos ofertados, seguindo uma distribuição e quantidades normais de todos os nutrientes (MARTINS et al., 2001).

Em relação às refeições observou-se, no atual estudo, que o lanche e a ceia apresentaram médias de DE significativamente superiores no lanche da tarde e na ceia. Esse resultado pode ser explicado pelo fato de que nessas duas refeições os pacientes costumam receber algum tipo de alimento processado, como biscoitos e bolos industrializados, que possuem um maior teor de gordura e açúcar, elevando assim a DE (NATIONAL INSTITUTE FOR HEALTH AND CARE EXCELLENCE, 2015), bem como observado no hospital do presente estudo.

Considerou-se como limitação do estudo, o fato da não inclusão de todos os líquidos como água, sucos e chás, que poderiam influenciar no volume das preparações e, consequentemente, na DE. Outra limitação a ser esclarecida é a exclusão de alguns tipos de dietas de algumas refeições, pois as mesmas eram produzidas pelo SND e os ingredientes eram acrescentados sem padronização exata. Logo, é recomendada 
a inclusão dessas preparações nas fichas técnicas de preparação, visando atualizar os dados da produção. Há ainda importância, o relato da dificuldade para discutir todas as variáveis encontradas, devido à falta de estudos atuais sobre o tema.

\section{CONCLUSÃO}

A DE encontrada no período analisado foi classificada como média e a diferença não significativa da DE entre os dias da semana, indicam um bom planejamento de cardápios, favorecendo a prevenção da obesidade e possibilitando uma melhora dos pacientes hospitalizados, por não receberem uma baixa DE diária. As diferenças significativas entre as refeições e entre as dietas sugerem uma revisão dos cardápios no SND. Mais estudos que avaliem a densidade energética das refeições em ambiente hospitalar devem ser conduzidos, com o objetivo de assegurar o cuidado nutricional hospitalar, concomitante ao bom andamento e padronização constante das atividades no SND.

\section{REFERÊNCIAS}

AGUIAR, Odaleia B. de; KRAEMER, Fabiana B.; MENEZES, Maria F. G. de. Gestão de pessoas em unidades de alimentação e nutrição. $1^{\text {a }}$ ed. Rio de Janeiro: Rubio, 2013.

BARTON, Arlene D. et al. A recipe for improving food intakes in elderly hospitalized patients. Clinical Nutrition, v. 19, n. 6, 2000. Disponível em: <http://www.sciencedirect. com/science/article/pii/S0261561400901493>. Acesso em: 20 fev. 2016.

CANELLA, Daniela S.; BANDONI, Daniel H.; JAIME, Patrícia C. Densidade energética de refeições oferecidas em empresas inscritas no Programa de Alimentação ao Trabalhador no Município de São Paulo. Revista de Nutrição, Campinas, v. 24, n. 5, 2011. Disponível em: http://www.scielo.br/scielo.php?script=sci_arttext\&pid=S1415-52732011000500005. Acesso em: 13 fev. 2016.

CARUSO, Lúcia; SIMONY, Rosana F.; SILVA, Ana L. N. D. da. Dietas hospitalares: uma abordagem na prática clínica. $1^{\mathrm{a}}$ reimpressão. São Paulo: Atheneu, 2004.

COX, David. N.; MELA, David J. Determination of energy density of freely selected diets: methodological issues and implications. International Journal of Obesity \& Related Metabolic Disorders, v. 24, n. 1, 2000. Disponível em: <http://web.a.ebscohost.com/abstract?direct=true\&profile=ehost\&scope=site \&authtype=crawler\&jrn$1=03070565 \& A N=8853343 \& \mathrm{~h}=\% 2 \mathrm{bW}$ bvDBuH1\%2fsPD471w5\%2foO 42756hyVw35ZmnlNDBm\%2bVaXHCvtzpCce9igLN8E1rgPZN6Yh0lt63h8ilArRn4dRQ\%3d\%3d\&crl=c\&resultNs=AdminWebAuth\&resultLocal=ErrCrlNotAuth\&crlhashurl=login. aspx $\% 3$ fdirect\%3dtrue\%26profile\%3dehost $\% 26$ scope $\% 3$ dsite\%26authtype\%3dcrawler\%26jrn1\%3d03070565\%26AN\%3d8853343>. Acesso em: 16 fev. 2016.

DIEZ-GARCIA, Rosa; PADILHA, Marina; SANCHES, Maísa. Alimentação hospitalar: proposições para a qualificação do Serviço de Alimentação e Nutrição, avaliadas pela 
comunidade científica. Ciência e Saúde Coletiva, v. 17, n. 2, 2012. Disponível em: <http:// www.scielosp.org/pdf/csc/v17n2/a20v17n2.pdf>. Acesso em: 13 fev. 2016.

DREWNOWSKI, Adam et al. Dietary Energy Density and Body Weight: Is There a Relationship? Nutrition Reviews, v. 62, n. 11, 2004. Disponível em: <http:// web.a.ebscohost.com/ehost/pdfviewer/pdfviewer?vid=14\&sid=ead3a868-df3b-44d9-91379cc38efb4825\%40sessionmgr4003\&hid=4109>. Acesso em: 20 fev. 2016.

ELLO-MARTIN, Julia; LEDIKWE, Jenny; ROLLS, Barbara. The influence of food portion size and energy density on energy intake: implications for weight management. American Journal of Clinical Nutrition, v. 82, n. 1, 2005. Disponível em: <http://ajcn. nutrition.org/content/82/1/236S.long>. Acesso em: 13 fev. 2016.

FERREIRA, Daiane; GUIMARÃES, Tessa G.; MARCADENTI, Aline. Aceitação de dietas hospitalares e estado nutricional entre pacientes com câncer. Einstein, v. 11, n. 1, jan./ mar. 2013. Disponível em: <http://www.scielo.br/scielo.php?script=sci_arttext\&pid=S167945082013000100008\&lang=pt>. Acesso em: 13 fev. 2015. DOI: 10.1590/S167945082013000100008

LEDIKWE, Jenny et al. Dietary energy density is associated with energy intake and weight status in US adults. American Journal of Clinical Nutrition, v.83, n. 6, 2006. Disponível em: <http://ajcn.nutrition.org/content/83/6/1362.long>. Acesso em: 01 nov. 2015.

LOTTENBERG, Ana M. P. Características da Dieta nas Diferentes Fases da Evolução do Diabetes Melito Tipo 1. Arquivo Brasileiro de Endocrinologia e Metabologia, v. 52, n. 2, 2008. Disponível em: <http://www.scielo.br/scielo.php?script=sci_arttext\&pid=S000427302008000200012\&lang=pt>. Acesso em: 13 fev. 2016.

MARTINS, Cristina et al. Manual de dietas hospitalares. 2a ed. São Paulo: Metha Ltda, 2001.

MENDES, Aline et al. Dietary energy density was associated with diet quality in Brazilian adults and older adults. Appetite, v. 97, 2016. Disponível em: <http://www.sciencedirect.com/ science/article/pii/S0195666315301021>. Acesso em: 20 fev. 2016.

MONSIVAIS, Pablo; DREWNOWSKI, Adam. Lower-Energy-Density Diets Are Associated with Higher Monetary Costs per Kilocalorie and Are Consumed by Women of Higher Socioeconomic Status. American Dietetic Association, 2009. Disponível em: <http://www.sciencedirect.com/science/article/pii/S0002822309001485>. Acesso em: 13 fev. 2016. doi:10.1016/j.jada.2009.02.002

NATIONAL CENTER FOR CHRONIC DISEASE PREVENTION AND HEALTH PROMOTION. Can eating fruits and vegetables help people to manage their weight? 2005. (Research to Practice Series, No 1). Disponível em: <http://www.cdc.gov/nccdphp/dnpa/ nutrition/pdf/rtp_practitioner_10_07.pdf>. Acesso em: 04 set. 2015.

NATIONAL INSTITUTE FOR HEALTH AND CARE EXCELLENCE. Preventing excess weight gain. mar. 2015, 55p. Disponível em: <http://www.nice.org.uk/guidance/ng7/ resources/preventing-excess-weight-gain-51045164485>. Acesso em: 13 fev. 2016.

NEPA-UNICAMP. Tabela Brasileira de Composição de Alimentos. Versão II. 2. ed. Campinas, SP: NEPA - UNICAMP, 2011. 161 p. Disponível em: <http://www.unicamp. 
br/nepa/taco/contar/taco_4_edicao_ampliada_e_revisada.pdf?arquivo=taco_4_versao_ ampliada_e_revisada.pdf >. Acesso em: 16 fev. 2016.

OLIVEIRA, Carolina S. de et al. Análise da densidade energética de preparações servidas em uma Unidade de Nutrição e Dietética. Nutrire. v. 35, n. 3, 2010. Disponível em: <http://files. bvs.br/upload/S/1519-8928/2010/v35n3/a1762.pdf>. Acesso em: 13 ago. 2015.

ORO, Gabrielle L.; HAUTRIVE, Tiffany P. Avaliação do cardápio do almoço oferecida a trabalhadores atendidos pelo Programa de Alimentação ao Trabalhador. e-Scientia. v. 8, n. 1, 2015. Disponível em: <http://revistas.unibh.br/index.php/dcbas/article/view/1454/936>. Acesso em: 19 fev. 2016.

RICARDO, Camila Z.; CLARO, Rafael M. Custo da alimentação e densidade energética da dieta no Brasil, 2008-2009. Caderno de Saúde Pública, v. 28, n. 12, 2012. Disponível em: <http://www.scielo.br/scielo.php?script=sci_arttext\&pid=S0102-311X2012001400013>. Acesso em: 04 set. 2015.

RODRIGUES, Aline Gouvea Martins; PROENÇA, Rossana Pacheco da Costa. Relação entre tamanho da porção de comida e ingestão alimentar: uma revisão. CERES, v. 6, n. 1, 2011. Disponível em: <http://www.e-publicacoes.uerj.br/index.php/ceres/article/ viewArticle/1968>. Acesso em: 01 set. 2015.

SILVA, Janiara D. Teor de energia, macronutrientes e minerais em dietas hospitalares orais prescritas a pacientes renais crônicos em tratamento conservador [trabalho de conclusão de curso - pós-graduação]. São Paulo: Universidade Federal de Ouro Preto. Escola de Nutrição, 2014. Disponível em: <http://www.repositorio.ufop.br/handle/123456789/4960>. Acesso em: 01 nov. 2015.

STELLA, Roberta H. Densidade energética: relação com variáveis demográficas, de estilo de vida, nutricionais e socioeconômicas em amostra representativa da população adulta do município de São Paulo [trabalho de conclusão de curso - pós-graduação]. São Paulo: Universidade de São Paulo. Faculdade de Saúde Pública, 2008. Disponível em: <http://www. teses.usp.br/teses/disponiveis/6/6133/tde-10092008-160343/pt-br.php>. Acesso em: 01 set. 2015.

TEIXEIRA, Suzana et al. Administração aplicada às Unidades de Alimentação e Nutrição. 1. ed. São Paulo: Atheneu, 2010.

WALLENGREN, Ola; LUNDHOLM, Kent; BOSAEUS, Ingvar. Diet energy density and energy intake in palliative care cancer patients. Clinical Nutrition, v. 24, 2005. Disponível em: <http://www.sciencedirect.com/science/article/pii/S0261561404001980?np=y >. Acesso em: 20 fev. 2016.

WALLENGREN, Ola; BOSAEUS, Ingvar; LUNDHOLM, Kent. Dietary energy density, inflammation and energy balance in palliative care cancer patients. Clinical Nutrition, v. 32, 2013. Disponível em: <http://www.sciencedirect.com/science/article/pii/ S0261561412001240>. Acesso em: 13 fev. 2016. 